\title{
Qualidade de vida profissional na saúde: um estudo em Unidades de Terapia Intensiva
}

\author{
Cláudia Gesserame Vidigal Mendes de Souza. Centro Universitário São Camilo \\ Gláucia Rosana Guerra Benute. Centro Universitário São Camilo \\ Maria Livia Tourinho Moretto. Universidade de São Paulo \\ Anna Sara Shafferman Levin. Universidade de São Paulo \\ Gracilene Ramos de Assis. Universidade de São Paulo \\ Maria Clara Padoveze. Universidade de São Paulo \\ Renata Desordi Lobo. Hospital Sírio-Libanês
}

\section{Resumo}

Objetivou-se avaliar a Qualidade de Vida Profissional por meio da análise de Satisfação por Compaixão, Burnout e Estresse Traumático Secundário em profissionais da saúde que atuam em Unidades de Terapia Intensiva, bem como verificar os fatores de risco para a ocorrência de Burnout e Estresse Traumático Secundário. O método adotado foi o estudo Survey em quatro UTIs de hospital universitário terciário da cidade de São Paulo. Foram utilizadas fichas de dados sociodemográficos e o instrumento ProQOL-BR. Verificou-se associação estatisticamente significativa entre Estresse Traumático Secundário e sexo $(p=0,03)$, e entre Satisfação por Compaixão, Burnout e Estresse Traumático Secundário $(p<0,01 ; p=0,002)$. Constatou-se, ademais, que existe desequilíbrio da qualidade de vida profissional dos participantes, e que os fatores de risco para Burnout e Estresse Traumático Secundário não estão relacionados com a área de atuação profissional, idade, escolaridade, estado civil ou renda.

Palavras-chave: saúde do trabalhador; unidades de terapia intensiva; fadiga por compaixão; profissionais da saúde.

\section{Abstract}

Professional quality of life in health: A study in Intensive Care Units. Objectives: To evaluate the Professional Quality of Life analizing Compassion Satisfaction, Burnout and Secondary Traumatic Stress among health professionals working in Intensive Care Units; to check the risk factors for the occurrence of Burnout and Secondary Traumatic Stress on those professionals. Method: Survey study conducted in four ICUs of tertiary university hospitals in São Paulo. Sociodemographic data was used along with the and ProQOL-BR tool. Results: There was a statistically relevant association between Secondary Traumatic Stress and gender $(p=0.03)$ and between Compassion Satisfaction, Burnout and Secondary Traumatic Stress $(p<0.01 ; p=0.002)$. Conclusion: It was found that there is instability in the professional quality of life of participants, and that the risk factors for Burnout and Secondary Traumatic Stress aren't related to professional area, age, education, marital status, or income.

Keywords: occupational health; intensive care units; compassion fatigue; health personnel.

\section{Resumen}

Calidad de vida profesional en salud: un estudio en Unidades de Cuidados Intensivos. Se objectiva evaluar la Calidad de Vida Profesional por medio del análisis de la Satisfacción por Compasión, Burnout y Estrés Traumático Secundario en el personal de la salud que trabajan en Unidades de Cuidados Intensivos y comprobar los factores de riesgo para la ocurrencia de burnout y estrés traumático secundario. El método adoptado es el estudio de encuesta en cuatro UCls de hospital universitario terciario en Sao Paulo. Se utilizó datos sociodemográficos y ProQol-BR. Se confirmó la asociación estadísticamente significativa entre Estrés Traumático Secundario y sexo $(p=0,03)$ y entre Satisfacción por Compasión entre Burnout y Estrés Traumático Secundario $(p<0,01 ; p=0,002)$. Se constató, además, que existe desequilibrio de la calidad de vida profesional de los participantes y que los factores riesgo para Burnout y Estrés Traumático Secundario no tiene relación con la práctica, edad, educación, estado civil o el ingreso profesional.

Palabras clave: salud laboral; unidades de cuidados intensivos; desgaste por empatía; personal de salud. 
As manifestações de sofrimento, decorrentes da condição de envolvimento emocional e afetivo do profissional que trabalha na assistência à saúde (PAS) ocasionados a partir do sofrimento e das dores dos pacientes a quem prestam assistência, têm recebido, na literatura da Psicologia, da Medicina e da Enfermagem, de forma geral, diversas nomeações, termos e definições como "Traumatização Vicária", "Traumatização Vicariante", "Traumatização Secundária", "Estresse Traumático Secundário", "Fadiga por Compaixão", "Fadiga da Compaixão", "Burnout" e "Contratransferência".

Essa variedade de termos e a forma de conceituá-los indica a falta de unanimidade entre pesquisadores interessados no fenômeno. Além disso, até o momento, não existem dados definitivos que sugerem que tais constructos são conceituadamente distintos (Craig \& Sprang, 2010; Lago \& Codo, 2013 Sabin-Farrel \& Turpin, 2003).

Sob variados termos, as manifestações decorrentes da condição de envolvimento emocional e afetivo de PAS ao sofrimento de pacientes a quem prestam assistência, têm sido bastante estudadas internacionalmente, mas ainda abordadas por poucos estudiosos brasileiros, como: Eizirik et al. (2006), Lago (2008), Lago e Codo (2010), Lago (2013), Lago e Codo (2013), Barbosa, Souza e Moreira (2014), Souza (2015), Boiças (2015), Silva (2015), Ribeiro, Cunha e Alvim (2015), Andrade et al. (2016), Santos (2018), Torres et al. (2018).

Uma forma de avaliar o envolvimento profissional e emocional associado ao cuidado ao paciente é a avaliação de "Qualidade de Vida Profissional" (QVP), proposta por Stamm (2010), que envolve "a qualidade que uma pessoa sente em relação ao seu trabalho como "helper"” (p. 8). Para a autora, os "helpers" envolvem profissionais de saúde, trabalhadores de serviço social, professores, advogados, policiais, bombeiros, membros do clero, agentes de companhias aéreas e de outras companhias de transportes, funcionários de limpeza de locais alvos de desastres e outros que oferecem assistência durante ou após um evento trágico.

Tal avaliação inclui dois aspectos; um positivo, denominado "Satisfação por Compaixão" (SC) e outro negativo, chamado de "Fadiga por Compaixão" (FC). A SC é caracterizada por sentimentos de satisfação pelo trabalho de assistência, envolvendo sentimentos positivos sobre a capacidade de ajudar, como sentir-se recompensado em seus esforços no trabalho e sentir que, com o trabalho, podem "fazer a diferença". Já a FC se refere às vivências negativas do trabalho de assistência e se desmembra em duas partes, a primeira diz respeito a sentimentos de exaustão, frustração e irritabilidade típicas do "Burnout" (BO), sendo definido como um esgotamento físico e psíquico que surge como reação ao estresse crônico no trabalho (Maslach, 2009). A segunda parte refere-se ao "Estresse Traumático Secundário" (ETS) ou "Trauma Secundário" (TS), caracterizado pelo medo e pelo trauma relacionado ao trabaIho de assistência em decorrência do contato do profissional com o sofrimento dos pacientes. Profissionais com ETS apresentam preocupações com pensamentos de pessoas a quem já ofereceram assistência, e relatam se sentirem "contaminados" pelo sofrimento e trauma dos assistidos. As características incluem a dificuldade para dormir, o esquecimento de coisas importantes e a incapacidade de separar vida pessoal da profissional, além de vivenciar o trauma e sofrimento de alguém que foi ajudado, a ponto de evitar atividades que possam acionar tais lembranças (Stamm, 2010, p. 8). (Figura 1).

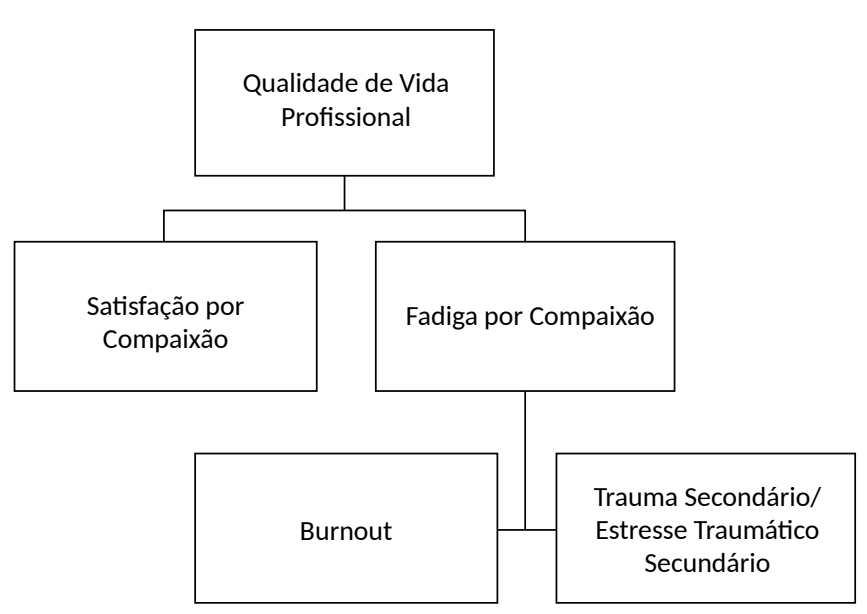

Figura 1. Diagrama da Qualidade de Vida Profissional (QVP) (Stamm, 2010, p. 8).

Portanto, a Qualidade de Vida Profissional se estabelece a partir do equilíbrio entre as experiências positivas e negativas, de modo que os sentimentos positivos prevaleçam sobre os negativos (Stamm, 2010).

Lago e Codo (2010), após terem investigado a origem de cada nomenclatura e definição na Literatura, explicado uma a uma e apontado as suas semelhanças e diferenças, apresentaram uma nova concepção dualista da empatia e da compaixão, distinguindo-a dos demais conceitos associados a ela, utilizando-se das expressões "Fadiga por Compaixão" e "Satisfação por Compaixão". Esses autores consideram que o processo empático que os seres humanos possuem é constituído 
por uma dimensão animal/biológica e outra simbólica/humana e "a compaixão advinda da capacidade de empatia também carrega essa dualidade, que é, a dualidade humana" (Lago, 2008). Para eles, a Fadiga por Compaixão é o "nome do processo no qual o profissional ligado ao atendimento de uma clientela que tem como demanda o sofrimento, torna-se fatigado, exausto física e mentalmente, devido ao constante contato com o estresse provocado pela compaixão". Já a Satisfação por Compaixão se caracteriza pela condição na qual o sujeito compadecido se percebe enquanto tendo sido responsável pela remissão da dor alheia (Lago \& Codo, 2010).

Em estudo realizado por Barbosa et al. (2014) que objetivou avaliar a QVP em 386 profissionais da saúde (74 médicos, 107 enfermeiros e 205 técnicos de enfermagem) atuantes em hospitais públicos nas cidades de João Pessoa e Campina Grande (PB), constatou-se, a partir do uso da validação do ProQol-IV de Barbosa e Souza (2013), que embora a maioria (79,8\%) tenha apresentado maior média em Satisfação por Compaixão, 22, 5\% da amostra apresentou indícios da FC. Os autores sugerem a realização de um debate entre os colaboradores e a gerência hospitalar a respeito das medidas e comportamentos que devem ser fortalecidos para elevar a SC e minimizar a FC. Identificaram também que a elevada carga horária de trabalho revelou-se um fator ameaçador da QVP e recomendam reorganização dos esquemas de horário, de acordo com as necessidades organizacionais e grupais. Além disso, apontaram limitações da pesquisa já que teve caráter exploratória e fora realizada em um contexto sociolaboral específico, composto por amostra não probabilística. Alertaram também para a importância de ampliar o estudo em médicos e a de realizar projetos futuros de intervenção que contemplem as variáveis analisadas e auxiliem as instituições hospitalares.

Com o objetivo de analisar a existência da Fadiga por Compaixão, da Síndrome de Burnout e da Satisfação por Compaixão em profissionais de enfermagem de Alagoas ( $n=750$ ), Santos (2018), em estudo quantitativo, descritivo e transversal, por meio do instrumento ProQol-BR (Lago \& Codo, 2013) identificou maiores médias para $\mathrm{BO}$ em profissionais solteiros e que afirmaram não ter uma patologia crônica diagnosticada. Constatou também que os colaboradores que possuíam segundo vínculo de trabalho na área hospitalar/pré-hospitalar apresentaram média significativa para FC.

Os fatores que contribuem para o desenvolvimento da FC parecem envolver aspectos subjetivos (aspectos da personalidade, educacional, experiência profissional e qualidade de vida pessoal) e institucionais (especificidade da função e reforma da saúde) (Pehlivan, 2018; Duarte \& Pinto-Gouveia, 2017). Torres et al. (2018), em estudo de revisão de literatura que objetivou analisar o que há de disponível na literatura sobre os fatores associados à Fadiga por Compaixão entre profissionais da saúde atuantes em hospitais, constataram que o desenvolvimento de $\mathrm{FC}$ entre profissionais da saúde está relacionado a fatores laborais e individuais associados às condições de trabalho, a fatores emocionais e psicológicos, e a fatores sociodemográficos. Em relação aos fatores sociodemográficos, verificaram que a idade tem um grande impacto na Qualidade de Vida Profissional (QVP), sendo os mais jovens a apresentarem maior risco de desenvolvimento da FC.

Também a respeito de fatores preditores de FC, Santos (2016) detectaram, em estudo quantitativo tranversal que compara 42 enfermeiros que trabalham em Cuidados Paliativos com 42 enfermeiros que trabaIham em Urgências, por meio de Escala de Satisfação das Necessidades Básicas (Sousa et al., 2012), ProQOL5 (Carvalho, 2011), Escala do Perfil de Atitudes Perante a Morte (Loureiro, 2010) e questionário sociodemográfico, que a Satisfação das Necessidades Básicas e atitudes perante a morte são fatores preditores do desenvolvimento de $\mathrm{BO}$ e de SC, porém não são da FC. De acordo com os autores, o estudo não encontrou diferenças entre os enfermeiros que atuam nestes dois locais e evidencia que quanto menor a atitude de evitamento da morte, mais elevada a Satisfação por Compaixão. Tal dado corrobora com as investigações de Figley (2002) e Stamm et al. (2002), a respeito das atitudes de compreensão dos profissionais e do elevado sentimento de empatia para com o doente provocarem Satisfação por Compaixão, e do aumento de BO e ETS em profissionais com atitudes negativas perante a morte, como medo e evitamento da morte.

O trabalho em UTIs envolve uma série de características específicas desse ambiente de trabalho, tais como a convivência diária dos profissionais e dos sujeitos doentes em situações de risco; a ênfase no conhecimento técnico-científico e na tecnologia para o atendimento biológico visando manter o ser humano vivo; a constante presença da morte; a ansiedade tanto dos sujeitos hospitalizados quanto dos familiares e trabalhadores de saúde; as rotinas muitas vezes rígidas e inflexíveis; e a necessidade de rapidez de ação no atendimento. Para Nascimento e Trentini (2004), a UTI pode ser considerada diferente das outras unidades de internação, uma vez que 
são ambientes conturbados, desconfortáveis, impessoais, sem privacidade, com isolamento social e com dependência da tecnologia e das aparelhagens múltiplas.

A Unidade de Terapia Intensiva (UTI) é considerada como a mais tensa, traumatizante e agressiva. O ambiente é insalubre e, dada a rotina de situações emergenciais e da concentração de pacientes críticos, o local de trabalho caracteriza-se como estressante, agressivo e emocionalmente comprometido para a equipe multiprofissional. (Rodrigues, 2012)

Diante do atual cenário a respeito da saúde mental dos profissionais no âmbito da assistência à saúde e tendo em vista que as características e especificidades das Unidades de Terapia Intensiva parecem favorecer a suscetibilidade dos profissionais da saúde a adoecimentos físicos e mental e os poucos estudos nacionais dedicados a investigar a Qualidade de Vida Profissional e Fadiga por Compaixão, este estudo busca contribuir para melhor compreensão deste fenômeno e se propõe a avaliar a Qualidade de Vida Profissional, por meio da análise de Satisfação por Compaixão, Burnout e Estresse Traumático Secundário (que compõem a Fadiga por Compaixão) em profissionais da saúde que atuam em Unidades de Terapia Intensiva, e verificar quais são os fatores de risco para a ocorrência de Burnout e Estresse Traumático Secundário nesses profissionais.

\section{Método}

\section{Amostra}

A amostra deste estudo foi constituída por conveniência com 168 profissionais da saúde, sendo 34 (18 médicos, três enfermeiros e 13 auxiliares e técnicos de enfermagem) da UTI de Moléstias Infecciosas, 52 (18 médicos, 14 enfermeiros e 20 auxiliares e técnicos de enfermagem) da UTI do Pronto Socorro, 41 (seis médicos, 11 enfermeiros e 24 auxiliares e técnicos de enfermagem) da UTI de Pneumologia e Clínica Médica e 41 (um médico, 16 enfermeiros e 24 auxiliares e técnicos de enfermagem) da UTI de Transplantes e Cirurgias do Fígado.

Trata-se de um estudo Survey para o qual foram convidados 183 profissionais que atuavam nas respectivas UTIs. Destes 183 profissionais, 15 não foram incluídos por estarem em horário de trabalho no período da coleta.

A coleta dos dados ocorreu entre os anos de 2013 e 2014 por meio de abordagem presencial aos PAS em seu local e horário de trabalho nos diferentes turnos (manhã, tarde e noite).

\section{Instrumentos}

Ficha de dados sociodemográficos. Para obtenção das informações sobre sexo, idade, profissão, tempo de atuação, escolaridade, estado civil, renda e unidade de trabalho.

ProQol-BR. Versão validada para o Brasil por Lago e Codo $(2008 ; 2013)$ da quarta versão do Professional Quality of Life Scale (ProQol-IV) de Stamm (2005). Lago e Codo (2013) apresentaram a validação semântica e psicométrica para o Brasil do ProQol-BR tendo como base a quarta versão do Professional Quality of Life Scale (ProQol-IV) de Stamm (2005). Por meio da validação semântica, os autores brasileiros asseguraram que as proposições contidas nas sentenças originalmente elaboradas em inglês sofreram o mínimo possível de distorção no processo de tradução e que o mesmo não cria sentenças de difícil compreensão para os brasileiros. Na validação psicométrica, os autores referem que, mesmo, após ser traduzido, o instrumento mantém boas qualidades psicométricas. Os resultados indicaram que a validação teve êxito em conseguir respeitar o sentido das sentenças e manter as propriedades psicométricas do instrumento (Lago \& Codo, 2013). A análise de consistência dos fatores foi avaliada por meio do alfa de Cronbach e apresentou índices de 0,81 para o primeiro fator, 0,83 para o segundo fator e 0,76 para o terceiro fator. (Lago \& Codo, 2013)

Trata-se de instrumento composto por 28 itens, que visa avaliar a QVP composta por aspectos positivos ("Satisfação por Compaixão") e aspectos negativos ("Fadiga por Compaixão"). Apesar de não ser instrumento de diagnóstico (já que a Fadiga por Compaixão não consta oficialmente na Classificação Internacional de Doenças (CID-10) ou no Manual Diagnóstico e Estatístico de Transtornos Mentais (DSM-V)), ele pode servir como "rastreador" de aspectos a serem investigados com o uso apropriado de procedimentos de diagnóstico. Por exemplo, uma pontuação alta nos itens de BO e ETS com uma baixa pontuação nos itens de Satisfação por Compaixão pode sinalizar a importância de avaliação psicológica para possível tratamento.

\section{Procedimentos}

O estudo, assim como o Termo de Consentimento Livre e Esclarecido (TCLE), foram aprovados pelo Comitê de Ética de um hospital universitário terciário da cidade de São Paulo, sob pareceres de números 623.825 e 18719, tendo sido autorizada a coleta de dados nas UTIs de Moléstias Infecciosas, Pronto-Socorro, Pneumologia e Clínica Médica, e Transplantes e Cirurgias do Fígado. 


\section{Análise das medidas}

Lago e Codo $(2008 ; 2013)$ realizaram a validação para o Brasil do ProQol-IV, no entanto, não publicaram parâmetros de interpretação dos dados do ProQol-BR, por isso, os dados foram analisados a partir das recomendações dispostas no Manual para avaliação do Professional Quality of Life Scale (ProQol-V) de Stamm (2010).

A utilização do "The Concise ProQOL Manual" de Stamm (2010) para analisar os resultados do ProQol-BR foi possível porque Stamm (2010), no manual, além de afirmar que a quinta versão é praticamente idêntica à quarta versão, tendo sofrido poucas mudanças gramaticais nas afirmativas, esclarece como realizar a conversão dos dados da quarta versão para a quinta, a partir da normalização dos dados, alcançada pela conversão dos resultados raw score em $\mathrm{t}$-score, que sempre tem média 50 e desvio padrão 10 (p. 31).

A aplicação do instrumento ProQol-BR com as orientações de Stamm (2010) para a interpretação dos dados permitiu saber a respeito do escore que um determinado PAS atingiu em cada sub-escala do instrumento. Desse modo, de acordo com o manual de Stamm (2010), dependendo do escore alcançado, ele poderá ser considerado baixo (quando o score for igual ou menor que 43), moderado (quando o score estiver entre 43 e 57) ou alto (quando o score for maior ou igual a 57). Por exemplo, um PAS pode apresentar um escore considerado alto na sub-escala de Satisfação por Compaixão (SC), um escore considerado moderado na sub-escala de Burnout (BO) e um escore considerado moderado na sub-escala de Estresse Traumático Secundário (ETS), o que comporá uma combinação específica a respeito de sua Qualidade de Vida Profissional (QVP).

Todos os dados foram inseridos no programa SPSS for Windows versão 16.0. Para a análise das tabelas de associação, utilizou-se o teste de qui-quadrado, aplicando-se a correção de Yates para continuidade. Nas situações em que o teste de qui-quadrado não pôde ser aplicado e, em se tratando de tabelas $2 \times 2$, foi utilizado o teste exato de Fisher. Adotou-se como nível de significância o valor 0,05 (alfa $=5 \%$ ). Com isso, níveis descritivos $(p)$ inferiores a esse valor foram considerados significantes $(p<0,05)$.

\section{Resultados}

Realizou-se a associação da SC, dividindo-se entre os níveis alto, moderado e baixo, com os dados sociodemográficos. Não foram constatadas significâncias estatísticas entre os dados, conforme mostra a Tabela 1.

Entre $\mathrm{BO}$ e as variáveis sociodemográficas, a associação tampouco demonstrou significância estatística, conforme os dados da Tabela 2.

$\mathrm{Na}$ associação entre ETS e as variáveis sociodemográficas, confirmou-se significância estatística entre ETS e sexo $(p=0,03)$, conforme os dados da Tabela 3 .

Já entre SC, BO e ETS, a associação apresentou significância estatística ( $p<0,01 ; p=0,002)$. Os resultados encontram-se apresentados na Tabela 4.

Tabela 1. Satisfação por Compaixão e Variáveis Sócio demográficas

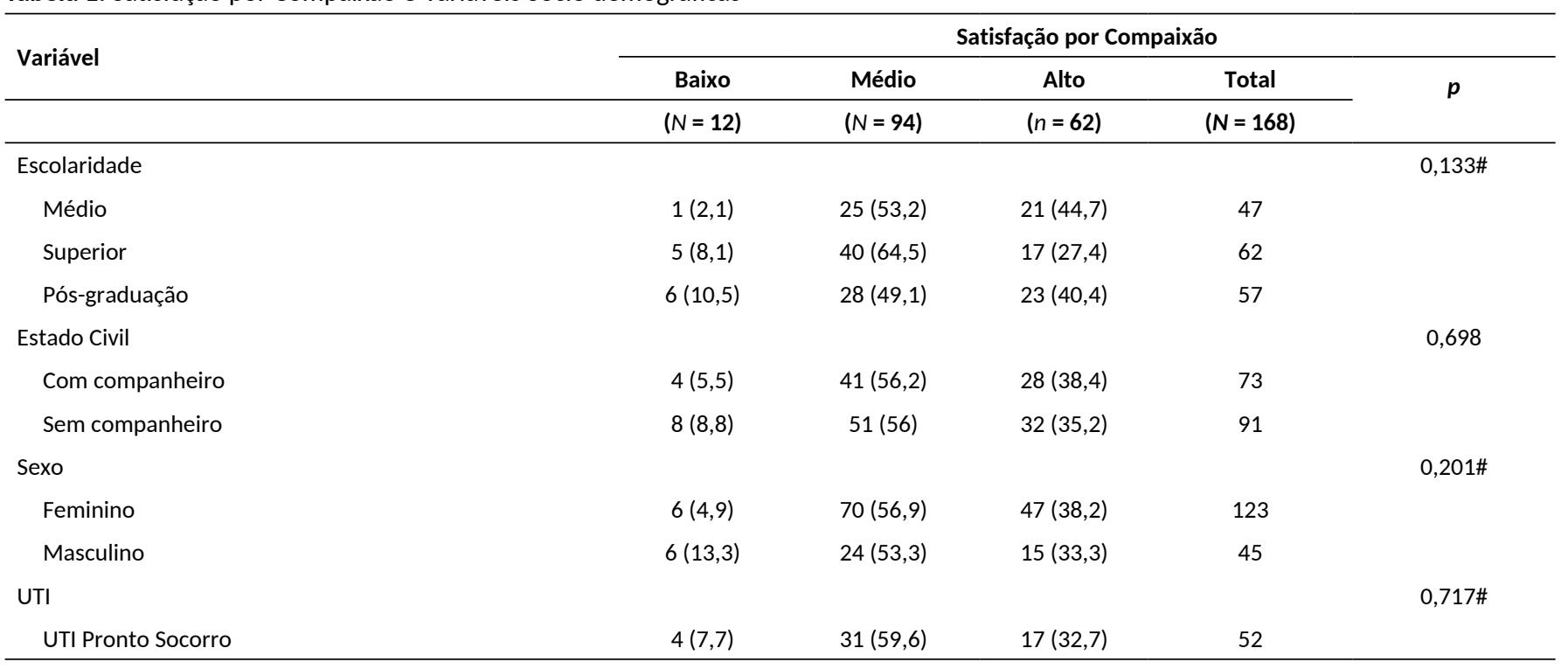


Qualidade de vida profissional na saúde: um estudo em unidades de terapia intensiva

Tabela 1. Continuação

\begin{tabular}{|c|c|c|c|c|c|}
\hline \multirow{2}{*}{ Variável } & \multicolumn{5}{|c|}{ Satisfação por Compaixão } \\
\hline & Baixo & Médio & Alto & Total & $p$ \\
\hline & $(N=12)$ & $(N=94)$ & $(n=62)$ & $(N=168)$ & \\
\hline UTI Moléstias Infecciosas & $3(8,8)$ & $21(61,8)$ & $10(29,4)$ & 34 & \\
\hline UTI Pneumo e Clínica Médica & $3(7,5)$ & $18(45)$ & $19(47,5)$ & 40 & \\
\hline UTI Transplantes e Cirurgias do Fígado & $2(4,9)$ & $23(56,1)$ & $16(39)$ & 41 & \\
\hline Profissão & & & & & $0,275 \#$ \\
\hline Médico & $3(7)$ & $28(65,1)$ & $12(27,9)$ & 43 & \\
\hline Enfermeiro & $5(11,4)$ & $25(56,8)$ & $14(31,8)$ & 44 & \\
\hline Auxiliar e Técnico de Enfermagem & $4(4,9)$ & $41(50,6)$ & $36(44,4)$ & 81 & \\
\hline
\end{tabular}

Nota. Teste qui-quadrado; \# Teste da razão de verossimilhanças.

Tabela 2. Burnout e Variáveis Sóciodemográficas

\begin{tabular}{|c|c|c|c|c|c|}
\hline \multirow{3}{*}{ Variável } & \multicolumn{4}{|c|}{ Burnout } & \multirow{3}{*}{$p$} \\
\hline & Baixo & Médio & Alto & Total & \\
\hline & $(N=22)$ & $(N=89)$ & $(N=57)$ & $(N=168)$ & \\
\hline Escolaridade & & & & & 0,372 \\
\hline Médio & $8(17)$ & $28(59,6)$ & $11(23,4)$ & 47 & \\
\hline Superior & $9(14,5)$ & $31(50)$ & $22(35,5)$ & 62 & \\
\hline Pós-graduação & $5(8,8)$ & $29(50,9)$ & $23(40,4)$ & 57 & \\
\hline Estado Civil & & & & & 0,13 \\
\hline Com companheiro & $12(16,4)$ & $42(57,5)$ & $19(26)$ & 73 & \\
\hline Sem companheiro & $10(11)$ & $44(48,4)$ & $37(40,7)$ & 91 & \\
\hline Sexo & & & & & 0,22 \\
\hline Feminino & $17(13,8)$ & $69(56,1)$ & $37(30,1)$ & 123 & \\
\hline Masculino & $5(11,1)$ & $20(44,4)$ & $20(44,4)$ & 45 & \\
\hline UTI & & & & & $0,786 \#$ \\
\hline UTI Pronto Socorro & $6(11,5)$ & $27(51,9)$ & $19(36,5)$ & 52 & \\
\hline UTI Moléstias Infecciosas & $6(17,6)$ & $17(50)$ & $11(32,4)$ & 34 & \\
\hline UTI Pneumo e Clínica Médica & $7(17,5)$ & $20(50)$ & $13(32,5)$ & 40 & \\
\hline UTI Transplantes e Cirurgias do Fígado & $3(7,3)$ & $25(61)$ & $13(31,7)$ & 41 & \\
\hline Profissão & & & & & 0,201 \\
\hline Médico & $4(9,3)$ & $21(48,8)$ & $18(41,9)$ & 43 & \\
\hline Enfermeiro & $3(6,8)$ & $24(54,5)$ & $17(38,6)$ & 44 & \\
\hline Auxiliar e Técnico de Enfermagem & $15(18,5)$ & $44(54,3)$ & $22(27,2)$ & 81 & \\
\hline
\end{tabular}

Nota. Teste qui-quadrado; \# Teste da razão de verossimilhanças.

Tabela 3. Estresse Traumático Secundário e Variáveis Sóciodemográficas

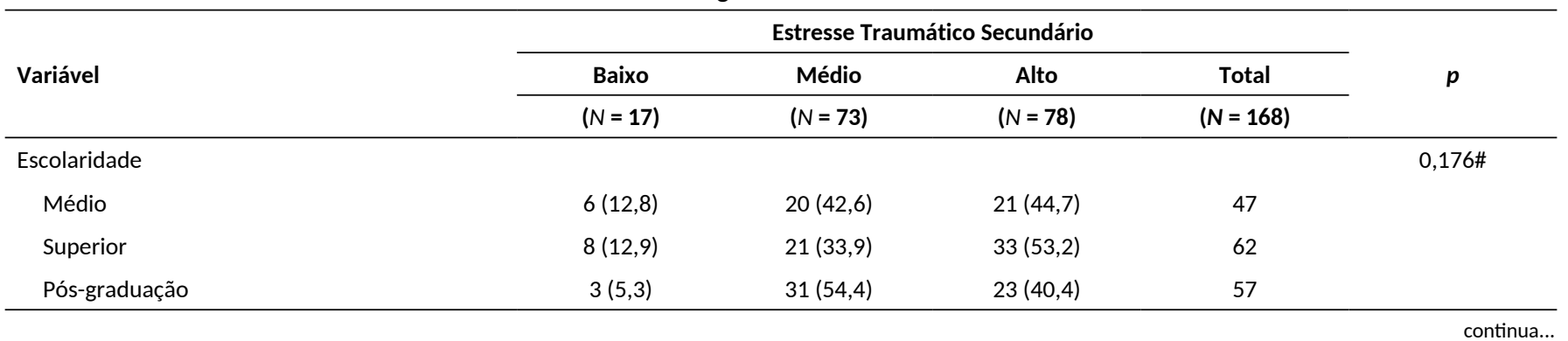


Tabela 3. Continuação

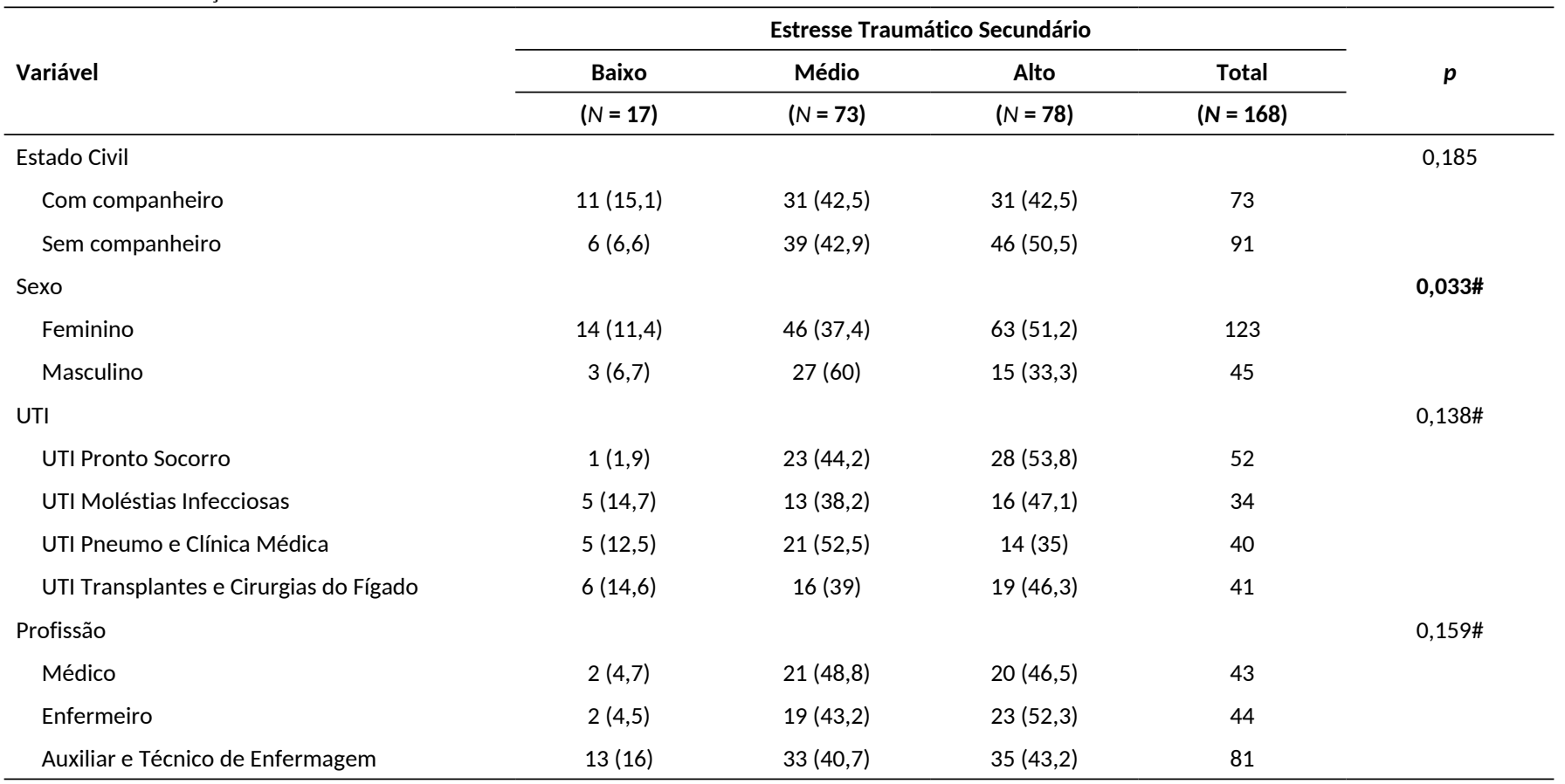

Nota. Teste qui-quadrado; \# Teste da razão de verossimilhanças.

Tabela 4. Associação entre Satisfação por Compaixão, Burnout e Estresse Traumático Secundário

\begin{tabular}{|c|c|c|c|c|c|}
\hline \multirow{3}{*}{ Variável } & \multicolumn{4}{|c|}{ Satisfação por Compaixão } & \multirow{3}{*}{$p$} \\
\hline & Baixo & Médio & Alto & Total & \\
\hline & $(N=12)$ & $(N=94)$ & $(n=62)$ & $(N=168)$ & \\
\hline Baixo & $0(0)$ & $5(3)$ & $17(10,1)$ & $22(13,1)$ & \\
\hline Médio & $1(0,6)$ & $53(31,5)$ & $35(20,8)$ & $89(53)$ & \\
\hline Estresse Traumático Secundário & & & & & 0,002 \\
\hline Baixo & $1(0,6)$ & $5(3)$ & $11(6,5)$ & $17(10,1)$ & \\
\hline Médio & $1(0,6)$ & $42(25)$ & $30(17,9)$ & $73(43,5)$ & \\
\hline Alto & $10(6)$ & $47(28)$ & $21(12,5)$ & $78(46,4)$ & \\
\hline
\end{tabular}

Nota. Teste da razão de verossimilhanças.

\section{Discussão}

O presente estudo constatou, entre os profissionais participantes, a existência de desequilíbrio da QVP uma vez que as experiências negativas (BO e ETS) que caracterizam a Fadiga por Compaixão (FC) prevaleceram sobre as experiências positivas (SC), indicando sofrimento destes profissionais, seja por envolvimento emocional e afetivo ao sofrimento e às dores dos pacientes (ETS), seja por esgotamento emocional e frustração com o trabalho e suas condições (BO).
A Fadiga por Compaixão ocorre frente à impossibilidade de se conseguir criar uma estratégia psíquica que faça com que o desgaste laboral - relacionado à insuficiência de recursos humanos e, em muitas instituições, com a carência de materiais - possa valer a pena, ou, ainda, quando o trabalho está estruturado de uma forma que não permite que o profissional crie tal estratégia e dê significado para todo o esforço empreendido. (Lago, 2013)

Há que se considerar, ainda, que além das vivências diárias de situações estressoras e de muito sofrimento decorrente do ambiente inóspito e das limitações do sistema de saúde (aspectos próprios do 
trabalho de assistência a pessoas adoecidas), ainda precisam manejar as questões emocionais que podem surgir do contato com os pacientes (e seus familiares) e das interferências de aspectos concernentes a vida pessoal no exercício profissional.

A fadiga, diferentemente do cansaço e da exaustão, que podem ser aliviados ou sanados por meio de estratégias restauradoras, é uma sintomatologia na qual o indivíduo não consegue encontrar alívio por meio do sono ou outras estratégias de descanso/relaxamento (Mota, Cruz, \& Pimenta, 2005; Ream \& Richarson, 1996). Assim, a fadiga pode ser compreendida como um sinal de alerta para reconhecimento dos limites por parte do organismo humano. Indica a necessidade de se buscar estratégias para o reestabelecimento da saúde do profissional, permitindo que os sintomas instalados possam ser revertidos. Não sendo obedecida, ela leva o profissional a um esgotamento físico e psíquico e à manifestação de alterações no funcionamento fisiológico das funções orgânicas (Leitão, 2008). A sintomatologia desencadeada pode ser: fadiga física (produzindo a incidência de dores lombares, dorsais, ombros e pescoço); fadiga mental (expresso sob a forma de cansaço mental, sensação de esgotamento) e fadiga nervosa (expressa sob a forma de manifestações de ansiedade, medo e frustração) (Campos \& David, 2011).

Lago (2013) destaca que a FC se associa mais diretamente aos quadros psiquiátricos e aspectos subjetivos do que com outros fatores estressores como turno de trabalho, qualidade do sono, alimentação, vínculos sociais ou longa jornada de trabalho.

Ramos, Souza, Goncalves, Pires e Santos (2014) apontaram em seu estudo que cada vez mais verifica-se, entre os profissionais da saúde, aumento de diagnósticos de transtornos mentais e reações como absenteísmo, agressão a clientes e/ou colegas e supervisores hierárquicos, não cumprimento de normas e rotinas da empresa, e o abandono da profissão. Apesar disso, as investigações apontam que as pesquisas sobre fadiga e saúde do trabalhador não têm considerado as questões emocionais envolvidas no trabalho (Lago, 2013).

Assim, este estudo evidencia a urgência de se propor cuidado a saúde emocional de PAS atuantes em UTIs já que a Fadiga por Compaixão aponta para um adoecimento mental e físico decorrente não somente de estresse crônico no trabalho, mas também das experiências subjetivas sofridas singulares de cada profissional da saúde ao vivenciar, na relação com os pacientes assistidos, o paradoxo de não se deixar envolver demais (empatia) nem de menos (antipatia) de seus pacientes, e de terem negligenciadas aspectos da subjetividade que ainda encontram poucos espaços para tratamento nas instituições de saúde.

Se o quadro de FC não for tratado, o objetivo dos profissionais da saúde, que é cuidar do doente, poderá ficar em risco uma vez que estes aspectos poderão "atuar" de forma negativa no momento do exercício da profissão, por meio do desenvolvimento inconsciente de mecanismos de defesas, como a negação da importância da pessoa, o distanciamento, a negação de sentimentos, o evitamento de decisões, diminuição do senso de responsabilidade profissional e iatrogenias.

Verificou-se, também, no presente estudo, que a prevalência de ETS foi maior entre as mulheres. Caracterizado por manifestação de medos e traumas relacionados ao trabalho de assistência em decorrência do contato do profissional com o sofrimento dos pacientes, o ETS em profissionais da saúde inclui preocupações com pensamentos de pessoas a quem ofereceram assistência, sentimento de estarem contaminadas pelo sofrimento e trauma dos outros, dificuldades para dormir, esquecimento de coisas importantes, dificuldades para separar vida profissional da vida pessoal e evitamento de certas atividades que podem trazer lembranças do sofrimento ou trauma da pessoa assistida. (Stamm, 2010).

Corroborando com os dados deste estudo, Carvalho (2011) identificou que os profissionais que apresentaram maiores níveis de ETS foram as mulheres $(22,5 \%)$ em comparação com os homens $(20,8 \%)$ ao realizar a primeira validação portuguesa da quinta versão do ProQol de Stamm em uma amostra de 24 profissionais da saúde (14 enfermeiros, sete médicos e três assistentes operacionais).

Com objetivo de estudar a Fadiga por Compaixão, a Satisfação no Trabalho e o Desajustamento Emocional em profissionais de cuidados pré-hospitalar/emergência e analisar as correlações entre elas, Manuelito (2016) verificou, além da faixa etária dos 44 aos 62 anos apresentar valores mais elevados para a SC, que existem diferenças significativas entre os gêneros, sendo os homens a referirem maior Satisfação no Trabalho, justificada pelo bom relacionamento com os colegas de profissão.

O autor Fonseca (2017), a partir de amostra de 87 enfermeiros de urgência hospitalar de adultos e análise da variação da FC em função das variáveis sociodemográficas e profissionais, por meio de questionário sociodemográfico e profissional, e ProQol-V de Stamm (2010) 
validado para a população portuguesa por Carvalho (2011), encontraram níveis altos de SC $(50,6 \%)$, BO $(54,0 \%)$ e ETS $(58,6 \%)$ verificando serem as mulheres as mais predispostas nas três subescalas. Enquanto a SC variou em função da idade e atividades de lazer, o BO variou em função da idade e o ETS da idade, sexo, tempo de experiência profissional e atividades de lazer.

Em pesquisa realizada por Boiças (2015) as mulheres evidenciaram níveis mais elevados de preocupação empática e expressão de sentimentos enquanto os homens relataram valores mais elevados de perseverança. Além disso, o uso de substâncias, o sentido da vida e o desconforto pessoal mostraram prever a FC.

Diante de tal resultado, que vai de encontro com a referida literatura, indaga-se se é possível levantar a hipótese de que as mulheres seriam mais suscetiveis ao desenvolvimento de ETS por se identificarem mais perante o sofrimento do outro, talvez por apresentarem mais sensibilidade e capacidade de empatia. Borges et al. (2019) sugerem existir associação entre as características do feminino, ligado à empatia e a ETS, no entanto destaca-se a necessidade de estudos que visem avaliar esta associação.

Avaliar as diferenças existentes nos processos de socialização emocional de homens e mulheres assim como as características atribuídas ao masculino e ao feminino podem auxiliar na compreensão da associação de ETS com o sexo feminino. As diferenças entre homens e mulheres não são determinadas apenas pelo sexo, mas influenciadas também pela classe social, pela cultura e pela educação, bem como por características individuais da personalidade, o que tem sido alvo de interesse por parte dos pesquisadores e está em constante debate e reflexão na sociedade. (Araújo, 2005). A cultura feminina tem sido descrita como aquela cuja atenção está focada nas outras pessoas e na qualidade dos relacionamentos, enquanto a cultura masculina é marcada pela valorização materialista e pela assertividade (Hofstede 1980 citado por Gondim, 2014).

Gondim et al. (2010) explicam que os estados afetivos, que abarcam emoções e sentimentos, são governados por regras socialmente compartilhadas que influenciam a intensidade com que devem ser sentidos, a situação a que devem ser dirigidos e sua duração. Além disso, "a avaliação de um sentimento apropriado a um contexto, além de levar em conta a situação (social), considera o que a pessoa está sentindo, o que ela pensa sentir e o que faz sobre o que sente (dimensão subjetiva)".
Além disso, é por meio da socialização, conforme as expectativas sociais, de gênero, status profissional, prestígio e posição social, familiar e profissional que a expressão de emoções e sentimentos é aprendida. "A rigor, controlamo-nos mutuamente por meio de expectativas sociais compartilhadas, nas quais se situam os estereótipos". (Gondim, Estramiana, Gallo, Vasconcellos, \& Bonfim, 2009).

Os estereótipos de gênero decorrem de processos cognitivos de atribuição e interferem significativamente em decisões que rebatem na qualidade das interações em ambientes de trabalho e são aprendidos no processo de socialização que varia de cultura para cultura (Gondim et al., 2009).

Uma outra possível explicação pode estar relacionada ao fato de, na amostra pesquisada, haverem mais mulheres $(n=123)$ do que homens $(n=45)$, indicando um viés do presente estudo. De todo modo, a importância da variável gênero ser investigada associada ao desenvolvimento da FC foi apontada por Lago (2013) em razão das atividades ligadas ao cuidado estarem historicamente associadas ao papel feminino. Além disso, segundo ele, do ponto de vista da profissão, "enquanto a medicina é uma profissão predominantemente masculina, a enfermagem é feminina" (p. 175).

Apesar de terem sido encontrados, em âmbito nacional, alguns artigos que também constataram maior sofrimento entre as mulheres no campo da FC, não foi localizado nenhum artigo que tenha tido como principal objetivo investigar associação entre FC ou QVP e a variável gênero por meio do controle de variáveis importantes como características de personalidade, alta prevalência feminina na enfermagem, possível maior predisposição das mulheres em expressarem suas emoções e sentimentos.

\section{Conclusão}

Esse estudo se propôs a avaliar a Qualidade de Vida Profissional por meio da análise de Satisfação por Compaixão, Burnout e Estresse Traumático Secundário em profissionais da saúde que atuam em UTIs, bem como verificar os fatores de risco para a ocorrência de Burnout e Estresse Traumático Secundário.

A análise da associação entre SC, BO e ETS revelou a existência de desequilíbrio da QVP uma vez que as experiências negativas (BO e ETS) prevaleceram sobre as experiências positivas (SC), indicando sofrimento dos profissionais participantes deste estudo, seja por 
envolvimento emocional e afetivo ao sofrimento e às dores dos pacientes (ETS), seja por esgotamento emocional e frustração com o trabalho e suas condições (BO).

Com relação aos fatores de risco para a ocorrência de BO e de ETS, verificou-se a não relação com área de atuação profissional, idade, escolaridade, estado civil ou renda, e que os profissionais com maior risco de desenvolver ETS foram do sexo feminino.

Com base nos resultados encontrados, recomenda-se que sejam construídos ou fortalecidos, nas instituições de saúde, espaços de atenção emocional aos profissionais da saúde, às mulheres em especial, que proporcionem a oportunidade de investigar as causas dos impactos nocivos em suas subjetividades e de tratamento a respeito das consequências de ser profissional da saúde e mulher, e de suas responsabilidades profissionais. Assim, poderão cuidar dos pacientes com o mínimo de interferência dos aspectos subjetivos nas responsabilidades profissionais, contribuindo para que a profissão seja exercida e não a atuação de aspectos de suas subjetividades. Além disso, sugere-se que sejam realizadas mais pesquisas a respeito dos aspectos psicológicos e da variável gênero em investigações relacionadas à qualidade de vida profissional e Fadiga por Compaixão.

\section{Referências}

Andrade, A. S., Antunes, N. A., Zanoto, P. A., Tiraboschi, G. A., Viana, P. V. B. A., \& Curilla, R. T. (2016). Vivências acadêmicas e sofrimento psíquico de estudantes de Psicologia. Psicologia: Ciência e Profissão, 36(4), 831-846. Recuperado de https://www.redalyc. org/pdf/2820/282048758005.pdf

Araújo, M. F. (2005). Diferença e igualdade nas relações de gênero: revisitando o debate. Psicologia Clínica, 17(2), 41-52. doi: 10.1590/ S0103-56652005000200004

Barbosa, S. C., Souza, S., \& Moreira, J. S. (2014). A fadiga por compaixão como ameaça à qualidade de vida profissional em prestadores de serviços hospitalares. Revista Psicologia: Organizações e Trabalho, 14(3), 315-323. Recuperado de http://pepsic.bvsalud.org/scielo. php?script=sci_arttext\&pid=S1984-66572014000300007\&lng= pt\&tlng=pt

Boiças, P. A. C. (2015). Fadiga por compaixão em voluntários na área da saúde: relações entre empatia, resiliencia e as estratégias de coping (Tese de Mestrado, Universidade Lusófona de Humanidades e Tecnologias, Lisboa, Portugal). Recuperado de http://recil. grupolusofona.pt/bitstream/handle/10437/7067/Tese\%20Final\%20 Patricia.pdf?sequence $=1$

Borges, E. M. N., Fonseca, C. I. N. S., Baptista, P. C.P., Queirós, C. M. L., Baldonedo-Mosteiro, M., \& Mosteiro-Diaz, M. P. (2019). Compassion fatigue among nurses working on an adult emergency and urgent care unit. Revista Latino-Americana de Enfermagem, 27, e3175. doi:10.1590/1518-8345.2973.3175

Campos, J. F., \& David, H. S. L. (2011). Avaliação do contexto de trabalho em terapia intensiva sob o olhar da psicodinâmica do trabalho. Revista da Escola de Enfermagem da USP, 45(2), 363-368. doi: 10.1590/ S0080-62342011000200009

Carvalho, P. R. C. (2011). Estudo da fadiga por compaixão nos cuidados paliativos em Portugal: tradução e Adaptação Cultural da Escala "Professional Quality of Life 5" (Dissertação de Mestrado, Universidade Católica Portuguesa, Porto, Portugal). Recuperado de http://hdl.handle.net/10400.14/8918

Craig, C. D., \& Sprang, G. (2010). Compassion satisfaction, compassion fatigue and burnout in a national sample of trauma treatment therapists. Anxiety, Stress \& Coping, 23(3), 19-339. doi: $10.1080 / 10615800903085818$

Duarte, J., \& Pinto-Gouveia, J. (2017). The role of psychological factors in oncology nurses' burnout and compassion fatigue symptoms. European Jounal of Oncology Nursing, 28,114-121. doi: 10.1016/j. ejon.2017.04.002

Eizirik, M., Schestatsky, S., Knijnik, L., Terra, L., \& Ceitlin, L. H. F. (2006). Contratransferência e trauma psíquico. Revista de Psiquiatria do Rio Grande do Sul, 28(3), 314-20. doi: 10.1590/ S0101-81082006000300010

Figley, C.R. (2002). Treating compassion fatigue. NewYork: Brunner-Routledge.

Fonseca, C. I. N. S. (2017). Fadiga por compaixão em enfermeiros de urgência hospitalar (Tese de Mestrado, Escola Superior de Enfermagem do Porto, Porto, Portugal). Recuperado de http://hdl. handle.net/10400.26/21098

Gondim, S. M. G., Estramiana, J. L. A., Gallo, I. S., Vasconcellos, C. M., \& Bonfim, M. C. (2009). Status profissional e gênero na atribuição intercultural de afetos no trabalho. RAM, Revista de Administração Mackenzie, 10(4), 75-99. doi: 10.1590/S1678-69712009000400005

Gondim, S.M.G., Estramiana, J.L.A., Luna, A.F., Souza, G.C., Sobral, L.C.S., \& Rego, C.C.A.B. (2010). Emoções e trabalho: estudo sobre a influência do status e do sexo na atribuição de afetos. Cademos de Psicologia Social do Trabalho, 13(2), 241-258. Recuperado de http://pepsic.bvsalud.org/ scielo.php?script=sci_abstract\&pid=S1516-37172010000200007\&lng= pt\&nrm=iso

Gondim, S. M. G., Morais, F. A., \& Brantes, C. A. A. (2014). Competências socioemocionais: fator-chave no desenvolvimento de competências para o trabalho. Revista Psicologia: Organizações e Trabalho, 14(4), 394-406. Recuperado de http://pepsic.bvsalud.org/scielo. php?script=sci_arttext\&pid=S1984-66572014000400006

Lago, K., \& Codo, W. (2010). Fadiga por Compaixão: o sofrimento dos profissionais em saúde. Petrópolis: Vozes.

Lago, K. C. (2008). Fadiga por compaixão: quando ajudar dói (Dissertação de Mestrado, Universidade de Brasília, Brasília). Recuperado de http://repositorio.unb.br/handle/10482/1291

Lago, K. C. (2013). Compaixão e trabalho: como sofrem os profissionais de saúde (Tese de Doutorado, Universidade de Brasilia, Brasilia). Recuperado de https://repositorio.unb.br/handle/10482/14514

Lago, K. C., \& Codo, W. (2013). Fadiga por compaixão: evidências de validade factorial e consistência interna do ProQol-BR. Estudos de Psicologia, 18(2), 213-221. Recuperado de http://www.scielo.br/pdf/ epsic/v18n2/v18n2a06.pdf

Leitão, I. M. T. A., Fernandes A. L., \& Ramos, I. C. (2008). Saúde ocupacional: analisando os riscos relacionados à equipe de enfermagem numa Unidade de Terapia Intensiva. Ciência, Cuidado e Saúde, 7(4), 476-84. doi: 10.4025/cienccuidsaude.v7i4.6630

Loureiro, L. M. J. (2010). Tradução e adaptação da versão revista da Escala de Avaliação do Perfil de Atitudes acerca da Morte (EAPAM). Revista de Enfermagem Referência, serllI(1), 101-108. Recuperado de http://www.scielo.mec.pt/scielo. php?script=sci_arttext\&pid=S0874-02832010000300011\&lng= pt\&tIng $=p t$ 
Manuelito, C. S. L. (2016). Fadiga por compaixão, satisfação no trabalho, stress, ansiedade e depressão em profissionais de cuidados pré-hospitalares/emergência (Dissertação de Mestrado, Universidade Lusófona de Humanidades e Tecnologias, Lisboa, Portugal). Recuperado de http //hdl.handle.net/10437/7061

Maslach, S. C. (2009). Comprendiendo el burnout. Revista Ciência \& Trabalho, 11(32), 37-43.

Mota, D. D. C. F., Cruz, D.A.L.M., Pimenta, C.A.M. (2005). Fadiga: uma análise do conceito. Acta paul. enferm. vol.18, n.3, 285-293. Recuperado de https://doi.org/10.1590/S0103-21002005000300009.

Nascimento, E. R. P., \& Trentini, M. (2004). O cuidado de enfermagem na unidade de terapia intensiva (UTI): teoria humanística de Paterson e Zderad. Revista Latino-Americana de Enfermagem, 12(2), 250-257. doi: 10.1590/S0104-11692004000200015

Pehlivan, T. (2018). Compassion fatigue: The known, unknown. Journal of Psychiatric Nursing, 9(2), 129-134. doi:10.14744/phd.2017.25582

Ramos, E. L., Souza, N. V. D. O., Gonçalves, F. G. A., Pires, A. S., \& Santos, D. M. (2014). Qualidade de vida no trabalho: repercussões para a saúde do trabalhador de enfermagem de terapia intensiva. Revista de Pesquisa: Cuidado é Fundamental (Online), 6(2), 571-583. doi: 10.9789/2175-5361.2014v6n2p571

Ream, E. Richardson, A. (1996) Fatigue: A Concept Analysis. International Journal of Nursing Studies, 33, 519-529. Recuperado de http://dx.doi.org/10.1016/0020-7489(96)00004-1

Ribeiro, M. G. S., Cunha, C. F., \& Alvim, C. G. (2015) Trancamentos de matrícula no curso de medicina da UFMG: sintomas de sofrimento psiquico. Revista Brasileira de Educação Médica, 40(4), 583-590. doi: 10.1590/1981-52712015v40n4e00282015

Rodrigues, T. D. F. (2012). Fatores estressores para a equipe de enfermagem da Unidade de Terapia Intensiva. Revista Mineira de Enfermagem, 16(3), 454-62. Recuperado de http://www.reme.org. br/artigo/detalhes/549

Sabin-Farrel, R., \& Turpin, G. (2003). Vicarious traumatization: Implications for the mental consequences of caring work? Clinical Psychology Review, 23(3), 449-480. doi: 10.1016/S0272-7358(03)00030-8

Santos, A. C. O. (2016). Satisfação das necessidades psicológicas básicas, satisfação e fadiga por compaixão e atitudes perante a morte em enfermeiros (Dissertação de Mestrado, Escola de Psicologia e Ciências da Vida, Lisboa, Portugal). Recuperado de http://recil. grupolusofona.pt/handle/10437/8392

Santos, A. G. (2018). Fadiga por compaixão, síndrome de burnout e a satisfação por compaixão em profissionais de enfermagem (Dissertação de Mestrado, Universidade Federal de Alagoas, Maceió). Recuperado de http //www.repositorio.ufal.br/handle/riufal/3726

Silva, A. T. C. (2015). Esgotamento profissional e depressão em profissionais da estratégia saúde da família no municipio de São Paulo (Tese de Doutorado, Faculdade de Medicina da Universidade de São Paulo, São Paulo). Recuperado de https://www.teses.usp.br/ teses/disponiveis/5/5137/tde-27102015-084632/pt-br.php

Sousa, S. S., Ribeiro, J. L. P., Palmeira, A. L., Teixeira, P. J., \& Silva, M $\mathrm{N}$ (2012). Estudo da basic need satisfaction in general scale para a língua portuguesa. Psicologia, Saúde \& Doenças, 13(2), 209 -219. Recuperado de http://www.scielo.mec.pt/pdf/psd/v13n2/v13n2a06. pdf

Souza, C. G. V. M. (2015). Investigação da fadiga elou satisfação por compaixão em profissionais da saúde nas práticas de controle de infecções relacionadas à assistência à saúde (Dissertação de Mestrado, Instituto de Psicologia da Universidade de São Paulo, São Paulo). doi: 10.11606/D.47.2015.tde-12112015-122237

SPSS for Windows (versão 16.0) (Programa de computador). Armonk, Nova lorque: IBM

Stamm, B. H. (2002). Measuring compassion satisfaction as well as fatigue: Developmental history of the Compassion Satisfaction and Fatigue Test. In C. R. Figley (Ed.), Psychosocial stress series, no. 24. Treating compassion fatigue (p. 107-119). Brunner-Routledge

Stamm, B. H (2005). The ProQOL Manual. Idaho State: Institute of Rural Health, University. Recuperado de http://www.compassionfatigue. org/pages/ProQOLManualOct05.pdf

Stamm, B. H. (2010). The Concise ProQOL Manual (2 ${ }^{\mathrm{a}}$ Ed. Pocatello) Recuperado de https://proqol.org/uploads/ProQOLManual.pdf

Torres, J.D. R.V., Cunha, F.O., Gonçalves, J.T.T., Torres, S. A. S., Barbosa, H. A., \& Silva, C. S. O. (2018). Fatores associados à fadiga por compaixão em profissionais de saúde, no contexto hospitalar: uma revisão na literatura. Temas em Saúde, 18(3), 2447-2131. Recuperado de http://temasemsaude.com/wp-content/uploads/2018/09/18310.pdf

Cláudia Gesserame Vidigal Mendes de Souza, Mestre em Ciências pelo Departamento de Psicologia Clínica da Universidade de São Paulo - USP, é Psicóloga do Centro Universitário São Camilo/ Pompéia. Endereço para correspondência: Centro Universitário São Camilo, Campus Pompéia. Rua Raul Pompéia - 144 Bloco C - Pompéia, CEP 05.025-010 - São Paulo, SP - Brasil. Telefones: (11) 3355-3929/

(11) 96381-3304. Email: claudia.vidigal@saocamilo-sp.br

Gláucia Rosana Guerra Benute, Doutora em Ciências pelo Departamento de Obstetrícia da Faculdade de Medicina da Universidade de São Paulo - USP, Pós-Doutora pela Universidade de São Paulo - USP, é Coordenadora do Curso de Psicologia do Centro Universitário São Camilo - CUSC. Email: psicologia@saocamilo-sp.br

Maria Livia Tourinho Moretto, Doutora em Psicologia Clínica pela Universidade de São Paulo - USP, é Professora Associada do Departamento de Psicologia Clínica do Instituto de Psicologia da

Universidade de São Paulo - USP. Email: liviamoretto@usp.br

Anna Sara Shafferman Levin, Doutora em Doenças Infecciosas e Parasitárias pela Faculdade de Medicina da Universidade de São Paulo - USP, é Professora titular da Faculdade de Medicina da Universidade de São Paulo - USP. Email: anna@usp.br 
Qualidade de vida profissional na saúde: um estudo em unidades de terapia intensiva

Gracilene Ramos de Assis, Doutora em Doenças Infecciosas e Parasitárias pela Faculdade de Medicina da Universidade de São Paulo - USP. Email: gracilenebio@gmail.com

Maria Clara Padoveze, Doutora em Clínica Médica, área básica, pela Faculdade de Ciências Médicas da Universidade Estadual de Campinas - UNICAMP, é Professora Associada - Livre-Docente da Universidade de São Paulo - USP. Email: padoveze@usp.br

Renata Desordi Lobo, Doutora em Ciências pelo Hospital das Clínicas da Faculdade de Medicina da Universidade de São Paulo - USP, é Enfermeira do Hospital Sírio-Libanês. Email: loborenata@gmail.com

Recebido em 21.nov.17 Revisado em 06.ago.19 Aceito em 29.nov.19 